\title{
Determination of phenolic composition and antioxidant activity in flowers and stems of Rumex tunetanus using RP-UHPLC-ESI-QTOF-MS
}

\author{
JoudaAbidia , SondaAmmar ${ }^{\mathrm{a}}$, Mohamed Bouaziz ${ }^{\mathrm{a} *}$.
}

${ }^{a}$ Laboratoired'Electrochimie et Environnement, EcoleNationaled'Ingénieurs de Sfax,Université de Sfax, BP1173, 3038 Sfax, Tunisia.

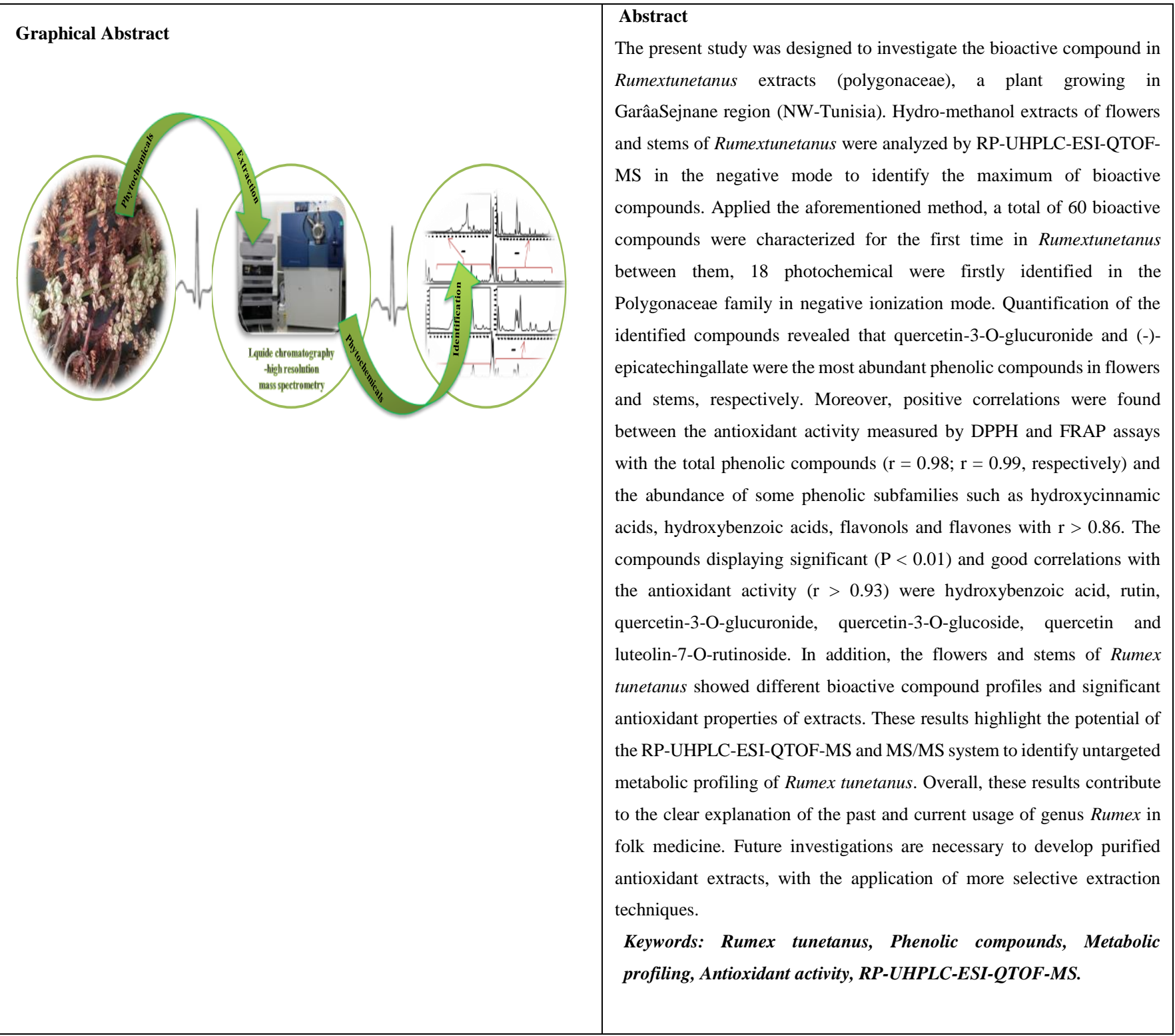

\section{Corresponding author:}

*Prof. Mohamed BOUAZIZ, Tel: +216 98667581 / Fax: +216 74674364 .

E-mail: mohamed.bouaziz@fsg.rnu.tn

\section{Introduction}

In response to the increased popularity and greater demand for medicinal plants, a recent study by world health organization claimed $80 \%$ dependency of word population on ethnomedicines[1]. 
increasing attention and it is growing phenomenally at the international level. However, the pharmacological evaluation of substances from plants is an established method for the identification of new compounds which can leads to the development of novel and safe medicinal agents in order to increase the dangerous side effects of synthetic molecules [2]. The genus Rumex represents one of the most important genera of Polygonaceae family, including approximately 200 species widely distributed in North American, European, African and Ausian countries [3]. Since very old times, differents species of rumex genus have been used for relief of symptoms of diseases. Much interest in Rumex species emanates from their long use in folk medicines as well as their pharmacological properties pharmacological activities such as anti-inflammatory, antidiuretic, antitumor, analgesic, antifungal and anti-viral activities [1].Large number of medicinal plants have been investigated for their antioxidant properties .Polyphenols are considered among the most important antioxidants in humain diet, and their presence in plant can protect consumers against oxidative stress, cardiovascular and chronic diseases [4].In fact, Rumex genus emerged as a good source of natural antioxidants. The major types of phenolic antioxidants found in Rumex genus include phenolic acids and their derivatives, namely gallic acid, dihydroxybenzoic acid, hydroxybenzoic acid and vanillic acid, and flavonoids, namely flavan-3ols(catechin, epicatechin and epicatechin gallate), flavonols (rutin, quercetin-3-O-glucoside, quercetin3-O-glucuronide, quercetin and isoramnetin), as well as condensed tannins as B-type procyanidin dimer and A-type procyanidin trimer[5]. Tunisia flora is known for its diversity of medicinal plant among them Rumex tunetanus. This plant was growing in the wet marshes of the Sejenane plain. Rumex tunetanus was never seen after its discovery in 1888, and more than 120 years after the discovery of this rumex, we find a large population of Rumex tunetanus in december 2009[6]. Therefore, as potential bioactive markers, the total phenolic content (TPC) and antioxidant capacity of flowers and stems of rumex tunetanus were firstly evaluated. Secondly, their phenolic profiles were extensively studied by ultra-high performance liquid chromatography (UHPLC) coupled with two detection systems, DAD and quadrupole time-of-flight (QTOF)-MS using electrospray ionization in negative ionization mode.

\section{Materials and Methods}

\section{Solvents and standards}

The solvents used for extraction were ultrapure water and methanol. All solvents used for HPLC-MS analysis were delivered by J.T Baker (Phillipsburg -USA). The reagents used to measure the TPC (total phenol content) and the antioxidant capacity were: Folin-Ciocalteau; sodium carbonate $\left(\mathrm{Na}_{2} \mathrm{CO}_{3}\right)$; 2,2diphenyl ,1-picrylhydrazyl (DPPH); 2,4,6-tri(2-pyridyl)-1,3,5-triazine (TPTZ) and ferric chloride $\left(\mathrm{FeCl}_{3} .6 \mathrm{H}_{2} \mathrm{O}\right)$; gallic acid, acetic acid $\left(\mathrm{C}_{2} \mathrm{H}_{4} \mathrm{O}_{2}\right)$; Ferric sulfate $\left(\mathrm{FeSO}_{4}\right)$; hydrochloric acid $(\mathrm{HCl})$; trihydrate sodium acetate $\left(\mathrm{C}_{2} \mathrm{H}_{3} \mathrm{NaO}_{2} .3 \mathrm{H}_{2} \mathrm{O}\right)$; sodium acetate; ascorbic acid. The phenolic standards used in our work were bought from Sigma-Aldrich (Saint-Louis -Missouri). The degree of purity of the standards was around $95 \%(\mathrm{w} / \mathrm{w})$. 
Flowers, stems and leaves from the Rumex tunetanus were collected from the Garâa Sejenane region (NW-Tunisia) in May 2014.The samples was harvested and transferred to the laboratory where they dried in the dark at room temperature $30^{\circ} \mathrm{C}$, and then they were finely ground prior to extraction. Flowers and stems were extracted using methanol/water 80:20 (v/v) as described elsewhere [7]. The residue were filtered with a syringe filter (regenerated cellulose, $0.45 \mu \mathrm{m}$ pore size) and stored at $-20{ }^{\circ} \mathrm{C}$ until future analysis. The extractions were repeated twice for each studied rumex tunetanus part.

\section{Total phenol content and antioxidant capacity assays}

The TPC of the extracts was determined in triplicate by the colorimetric assay using the Folin-Ciocalteau reagent as reported by Ammar et al.[8]. The DPPH assay was based on the method described by Gargouri et al.[9]. The FRAP assay was conducted following the method described by Ammar et al. [10].

\section{Mass spectrometry-based analyses}

The analyses were made with an Agilent 1200 series rapid resolution (Palo Alto, CA, USA). The system was coupled with a DAD and a 6540 Agilent ultra-high-definition (UHD) accurate-mass QTOF LC/MS, which was equipped with Agilent Dual Jet Stream electrospray ionization (Dual AJS ESI) interface. The detail of negative ionization modes was described by Abidi et al [7].

\section{Databases}

In addition of consulting literature, the following databases were used to retrieve chemical information: PubChem (http://pubchem.ncbi.nlm.nih.gov), ChemSpider (http://www.chemspider.com), SciFinder Scholar (https://scifinder.cas.org), Reaxys (http://www.reaxys.com), Phenol-Explorer (www.phenolexplorer.eu) and KNApSAcK Core System (http://kanaya.naist.jp/knapsack jsp/top.html).MassBank and Metlin Metabolite Database were used to check fragmentation patterns in some cases.

\section{Results and Discussion}

Total phenolic content (TPC) and antioxidant activity of the Rumex tunetanus flowers and stems

The determination of TPC of the extracts was performed by the Folin-Ciocalteu assay and calculated as gallic acid equivalents and is listed in Table 1. Rumex tunetanus flowers extract was found to contain higher total phenolic contents (146.20 mg GAE/g extract) as compared to Rumex tunetanus stems extract (118.88 mg GAE/g extract).

The extracts were evaluated for their antioxidant /radical scavenging activity by DPPH and FRAP. The results of percentage scavenging are depicted in Table 1. In general, both of these two analyses showed that the hydro-methanolic extract of Rumex tunetanus exhibited good antioxidant activities. According to the aforementioned results for TPC, the flowers showed higher antioxidant activity values than stems by the two assayed methods. In fact, our results have indicated that the DPPH value of Rumex tunetanus flowers and stems extracts are in accordance with those obtained by Yousef et al. [11] forRumex tuberosus flowers $(0,65 \mathrm{mg} / \mathrm{ml}$ of extract) and stems $(0,69 \mathrm{mg} / \mathrm{ml}$ of extract).

\section{Characterization strategy and fragmentation pattern study}


RP-HPLC-DAD-QTOF-MS using ESI in the negative mode. This is the commonest ionization source and mode used to identify the phenolic and non-phenolic compounds in Rumex tunetanus flowers and stems. Using this methodology, our characterization steps could be basically summarized by a targeted searching of previously, an untargeted analysis and a predictive study of unreported phenolic structures based on all the spectrometric data obtained by the detection techniques applied (Figure 1).

Thanks to the methodology proposed in the present study, a total of 60 metabolites (60 in flowers and 51 in stems (Table 2) have been tentatively identified, including sugars (1), organic acid (3), hydroxybenzoic acids (8), hydroxycinnamic acids (1), flavonoids (28) as well as tannins (19). These results exemplify that RP-HPLC-DAD-QTOF-MS is useful to detect and characterize novel chemical structures.

\section{Comparison between flowers and stems}

Table 1 shows the qualitative differences between flowers and stems: presence (+) or absence (-) of the characterized compounds. In brief, more than $90 \%$ of the compounds were in common in both extracts. Moreover, as summary Figure 2 depicts these differences in terms of number of compounds found in each phenolic class. Interestingly, flavonoids and their glycoside derivatives were characterized in the negative ionization mode as a more widely spread phenolic group of Rumex tunetanus samples with 28 compounds. To the best of our knowledge, this is the first time that dihydroxybenzoic acid hexoside (8), syringic acid hexoside (12) and tri- $O$-methoxyellagic acid (51) have been identified in Polygonaceaefamily.

\section{Conclusions}

Globally, these results exemplify the usefulness of RP-HPLC-DAD-QTOF-MS to perform characterization studies, such as that described here. In this way, 60 phenolic compounds were characterized in flowers and 51 in stems of rumex tunetanus which were classified into twelve compound families that possess potent antioxidant activities. Flavonols, flavanols, flavones, hydroxybenzoic acids and condensed tannins were the most representative groups in both samples by using the relative amounts of the phenolic compounds. In addition, the antioxidant properties of extracts were evaluated in terms of single electron transfer as DPPH and FRAP assay and Rumex tunetanus flowers exhibited significantly higher antioxidant activities than the stems. Besides, the isolation of pure compounds with pharmacological activities holds significance in contemporary and future research. Recently, the plant extract was also being used by the researchers to produce nanoparticles [2]. Therefore, there is huge room for research in these directions.

\section{References}


polygonaceae family. 2017;6(6):587-589. doi:10.1016/j.jep.2015.09.001.

[2] Shaikh S, Shriram V, Srivastav A, Barve P, Kumar V. A critical review on Nepal Dock ( Rumex nepalensis): A tropical herb with immense medicinal importance. 2018;11(7):405-414. doi:10.4103/1995-7645.237184.

[3] Islam MS, Iwasaki A, Suenaga K. all Aspects of Plant Biology Evaluation of phytotoxic potential and identification of phytotoxic compounds in Rumex maritimus. Plant Biosyst - An Int J Deal with all Asp Plant Biol. 2017;3504(July):1-6. doi:10.1080/11263504.2017.1338630.

[4] Ouerghemmi S, Sebei H, Siracusa L, et al. Comparative study of phenolic composition and

\begin{tabular}{cccc}
\hline Extract & TPC & DPPH & FRAP \\
\hline Flowers & $146.2 \pm 1.86$ & $0.72 \pm 0.230$ & $528.24 \pm 32.36$ \\
Stems & $118 \pm 1.05$ & $0.68 \pm 0.230$ & $307.04 \pm 54.36$ \\
\hline
\end{tabular}

antioxidant activity of leaf extracts from three wild Rosa species grown in different Tunisia regions : Rosa canina L ., Rosa moschata Herrm . and Rosa sempervirens L. Ind Crop Prod. 2016;94:167-177. doi:10.1016/j.indcrop.2016.08.019.

[5] El-hawary SA, Sokkar NM, Ali ZY, Yehia MM. A Profile of Bioactive Compounds of Rumex. 2011. doi:10.1111/j.1750-3841.2011.02370.x.

[6] Ghrabi-Gammar Z, Muller SD, Rouissi M, et al. Rumex tunetanus (Polygonaceae): Rediscovery of an endangered tunisian endemic. Phytotaxa. 2017;296(2):118-130. doi:10.11646/phytotaxa.296.2.2.

[7] Abidi J, Ammar S, Brahim S Ben, Ghrabi-gammar Z, Bouaziz M. Journal of Pharmaceutical and Biomedical Analysis Use of ultra-high-performance liquid chromatography coupled with quadrupoletime-of-flight mass spectrometry system as valuable tool for an untargeted metabolomic profiling of Rumex tunetanus flowers and stems and contribution to the antioxidant activity. 2019;162:66-81. doi:10.1016/j.jpba.2018.09.001.

[8] Ammar S, Contreras M, Belguith-hadrich O, Segura-carretero A, Bouaziz M. Food \& Function compounds and contribution to the antioxidant. 2015:3663-3677. doi:10.1039/c5fo00837a.

[9] Gargouri B, Ammar S, Zribi A, Mansour A Ben, Bouaziz M. Effect of growing region on quality characteristics and phenolic compounds of chemlali extra-virgin olive oils. Acta Physiol Plant. 2013;35(9):2801-2812. doi:10.1007/s11738-013-1312-z.

[10] Sonda A, Akram Z, Boutheina G, Flamini G, Mohamed B. Effect of addition of olive leaves before fruits extraction process to some monovarietal tunisian extra-virgin olive oils using chemometric analysis. J Agric Food Chem. 2014;62(1):251-263. doi:10.1021/jf404395x.

[11]A.A.D. Yousef Ghasemi, Mohammad Ali Ebrahimzadeh, Sareh Rezaei, Mahdieh Taheri.Seyyedeh Fatemeh Hashemian, In vitro antibacterial activity and the explant type and extraction solution biochemical properties of Rumex tuberosus effect of, Int. J. Life Sci. Pharma Res. 6 (2016) L7L13.

Table 1Total phenolic content (TPC) (mg of GAE/g of the extract) determination and antioxidant capacities of the studied Rumextunetanusflowers and stems parts as measured by DPPH (mg/ml of extract) and FRAP (mmol eq. $\mathrm{FeSO}_{4} / \mathrm{g}$ of the extract) antioxidant assays. 


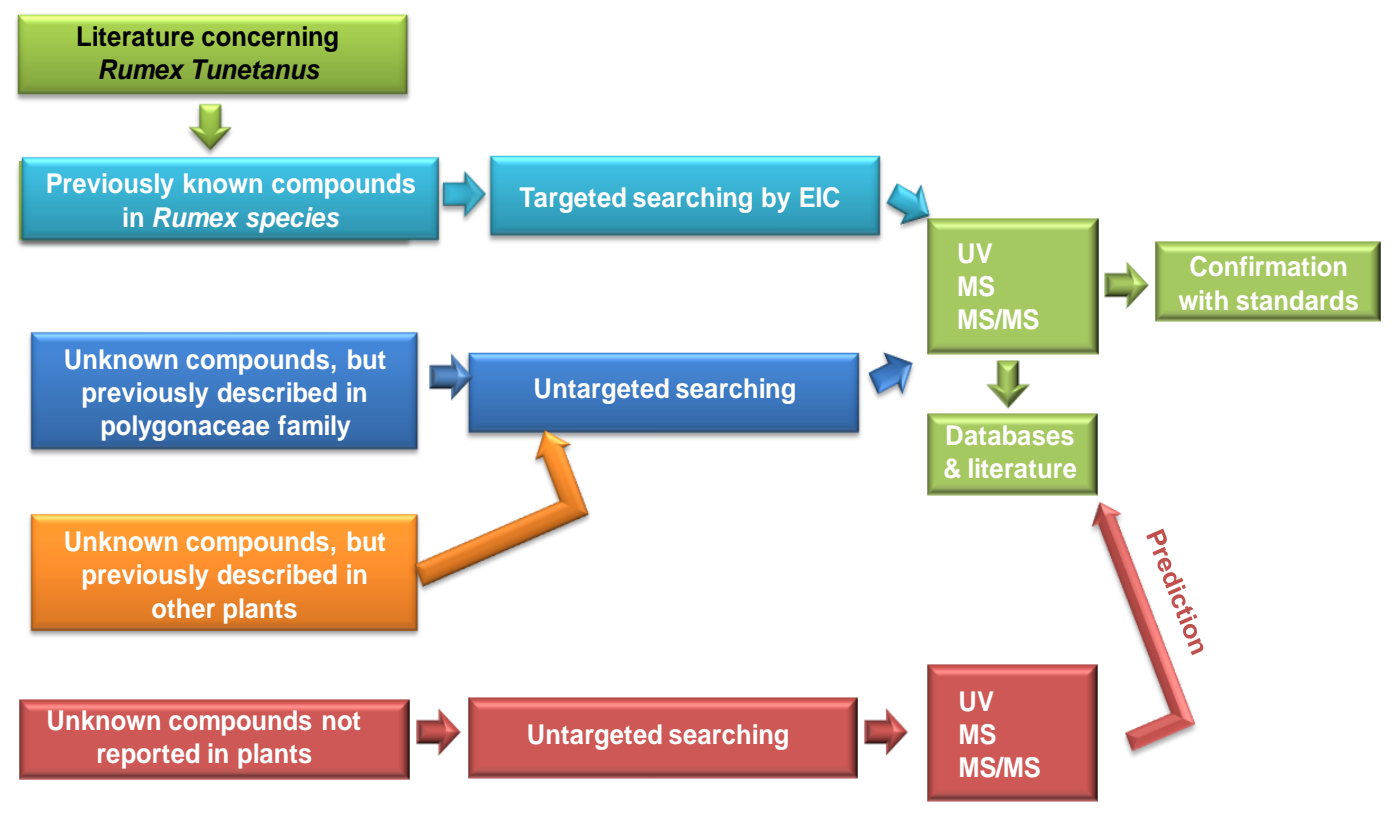

Figure 1: The strategy for deducing of the most possible structures of Rumex tunetanus bioactive compounds 

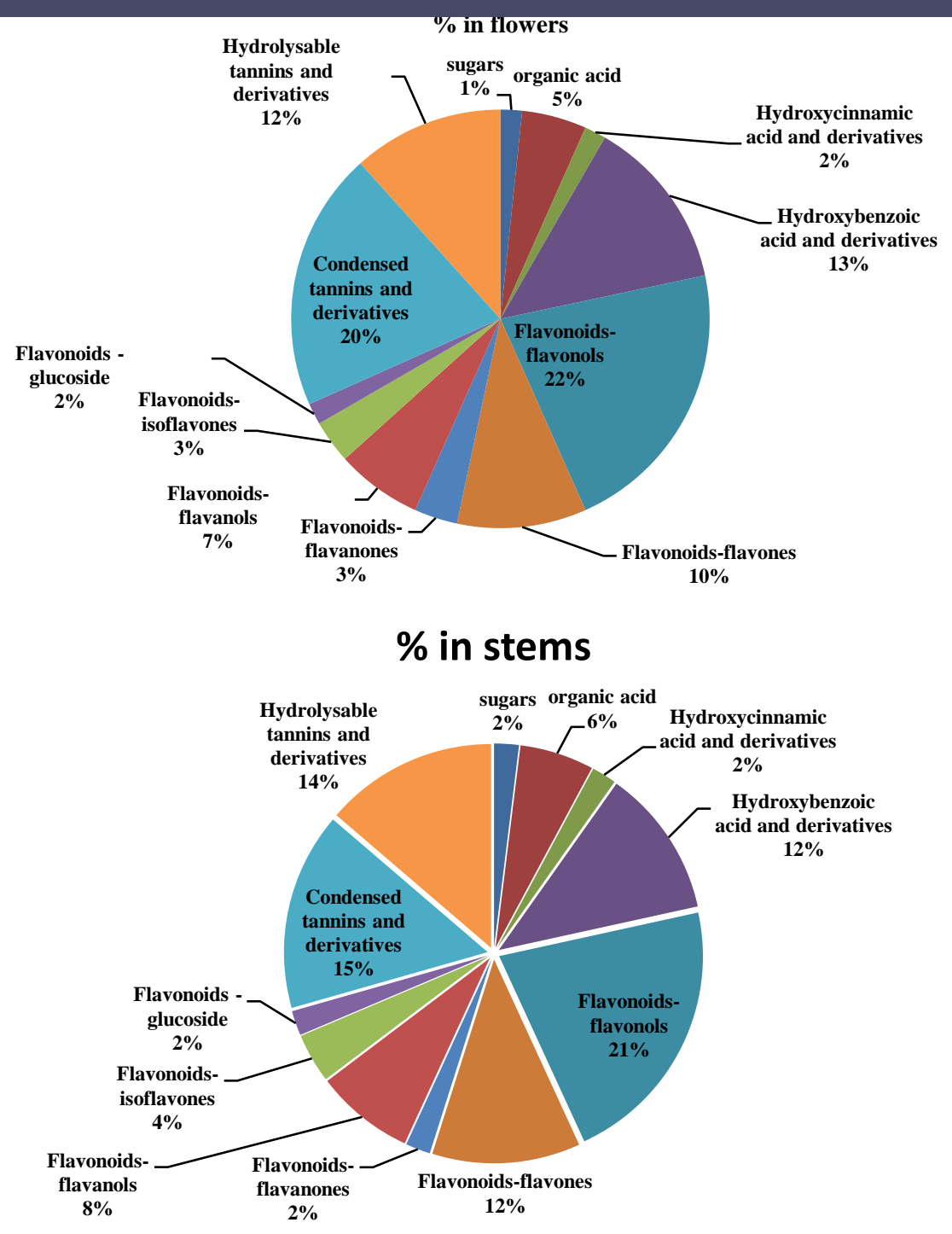

Figure2. Various compounds found in each phenolic class of flowers and stems of rumex tunetanus

Table 2. Compounds characterized by HPLC-DAD-QTOF-MS in Rumex tunetanus flowers and stems 


\begin{tabular}{|c|c|c|c|c|c|c|c|c|c|c|c|}
\hline $\mathbf{N}^{\circ}$ & $\mathbf{R T}^{\mathrm{a}}(\min )$ & $\begin{array}{c}\text { Exp. }^{\text {a }} \mathbf{m} / \mathbf{z} \text { for } \\
{[\mathrm{M}-\mathrm{H}]^{-}}\end{array}$ & Proposed compound & Preser & ce in & $\mathbf{N}^{\circ}$ & RTa (min) & Exp.a m/z for [M-H]- & Proposed compound & Presen & cee in \\
\hline & & & & Flowers & stems & & & & & Flowers & Stems \\
\hline Sugars & & & & & & 58 & 36.81 & 329.0673 & Tricin II & + & + \\
\hline 1 & 1.52 & 341.1116 & Sucrose & + & + & 60 & 39.70 & 329,0643 & Tricin III & + & + \\
\hline Organic Acid & & & & & & $\begin{array}{l}\text { Flavonoids- } \\
\text { flavanones }\end{array}$ & & & & & \\
\hline 2 & 1.59 & 191.0542 & Quinic acid I* & + & + & 50 & 30.69 & 287.0562 & Eriodictyol* & + & - \\
\hline 4 & 1.82 & 191.0538 & Quinic acid II & + & + & 55 & 34.39 & 271.0605 & Naringenin* & + & + \\
\hline 5 & 1.97 & 191.0183 & Citric acid* & + & + & $\begin{array}{l}\text { Flavonoids- } \\
\text { flavanoles }\end{array}$ & & & & + & + \\
\hline $\begin{array}{l}\text { Phenolic acid } \\
\text { (Hydroxycinna } \\
\text { mic acid and } \\
\text { derivatives ) }\end{array}$ & & & & & & 16 & 17.03 & 289.0727 & $(+)$-Catechin*c & + & + \\
\hline 19 & 17.94 & 355.1028 & Ferulic acid hexoside & + & + & 23 & 19.53 & 289.0727 & (-)-Epicatechin*c & + & + \\
\hline $\begin{array}{l}\text { Phenolic acid } \\
\text { (Hydroxybenzoi } \\
\text { c acids and } \\
\text { derivatives) }\end{array}$ & & & & & & 33 & 23.96 & 441.0824 & $(-)$-Epicatechingallate ${ }^{c}$ & + & + \\
\hline 7 & 3.75 & 169.0137 & Gallic acid* & + & + & 47 & 27.37 & 287.0556 & Fustin $^{\mathrm{b}}$ & + & + \\
\hline 8 & 4.43 & 315.0725 & Dihydroxybenzoic acid hexosideb & + & - & $\begin{array}{l}\text { Flavonoids- } \\
\text { isoflavones }\end{array}$ & & & & & \\
\hline 9 & 8.88 & 153.0186 & Dihydroxybenzoic acid hexosideb & + & + & 57 & 36.57 & 299.0568 & $\begin{array}{l}\text { 7-Methoxy 2'-hydroxy genistein } \\
\text { (cajanin)b }\end{array}$ & + & + \\
\hline 10 & 12.14 & 137.024 & Hydroxybenzoic acid I* & + & + & 59 & 37.38 & 359.078 & $\begin{array}{l}\text { 5.7.4 -trihydroxy- } 6.3 .5- \\
\text { trimethoxyisoflavone (Irigenin) }\end{array}$ & + & + \\
\hline 11 & 12.96 & 137.0236 & Hydroxybenzoic acid II & + & + & $\begin{array}{l}\text { Flavonoids- } \\
\text { glucoside }\end{array}$ & & & & + & + \\
\hline 12 & 13.94 & 359.0976 & Syringic acid hexosideb & + & + & 46 & 27.25 & 591.1357 & $\begin{array}{l}\text { Flavonoid glucoside-HMG } \\
\text { conjugateb }\end{array}$ & + & + \\
\hline 25 & 20.43 & 167.0347 & Vanillic acid* & + & - & $\begin{array}{l}\text { Condensed } \\
\text { tannins and } \\
\text { derivatives }\end{array}$ & & & & + & + \\
\hline 51 & 31.38 & 343.0429 & Tri-O-methoxyellagicacidb & + & + & 13 & 15.82 & 577.1334 & B-type procyanidin dimer $\left(I^{c}\right.$ & + & + \\
\hline $\begin{array}{l}\text { Flavonoids- } \\
\text { flavonols }\end{array}$ & & & & & & 14 & 16.34 & 577.1334 & B-type procyanidin dimer (II) ${ }^{c}$ & + & - \\
\hline 34 & 24.11 & 463.0881 & Quercetin-3-O-glucoside I*c & + & + & 26 & 20.48 & 729.1472 & B-type procyanidin dimer gallate I & + & - \\
\hline 35 & 24.33 & 477.0656 & Quercetin-3-O-glucuronide* & + & + & 27 & 20.72 & 863.1813 & A-type procyanidintrimer & + & + \\
\hline
\end{tabular}




\begin{tabular}{|c|c|c|c|c|c|c|c|c|c|c|c|}
\hline 36 & 24.44 & 463.0884 & Quercetin-3-O-glucoside Iic & + & - & 28 & 21.09 & 729.1465 & B-type procyanidin dimer gallate II & + & - \\
\hline 39 & 25.31 & 607.1306 & $\begin{array}{l}\text { Quercetin-3-[6"-(3-hydroxy-3- } \\
\text { methylglutaryl)][-hexosideb }\end{array}$ & + & + & 29 & 21.47 & 577.1363 & B-type procyanidin dimer $(\mathrm{V}) \mathrm{c}$ & + & + \\
\hline 40 & 25.56 & 599.1050 & $\begin{array}{l}\text { Quercetindihydroxybenzoylhexosi } \\
\text { deb }\end{array}$ & + & + & 30 & 21,63 & 729.1455 & $\begin{array}{l}\text { B-type procyanidin dimer gallate } \\
\text { III }\end{array}$ & + & + \\
\hline 43 & 26.44 & 505.099 & Quercetin-3-O-hexosyl-6"-acetate & + & - & 37 & 24.8 & 729.1464 & $\begin{array}{l}\text { B-type procyanidin dimer gallate } \\
\text { IV }\end{array}$ & + & - \\
\hline 45 & 27.71 & 625.1183 & Quercetin-O-dihexoside & + & + & 44 & 26.61 & 729.1473 & B-type procyanidin dimer gallate & + & + \\
\hline 49 & 29.67 & 639.1339 & Quercetin-O-feruloylhexosideb & + & + & 48 & 28.13 & 881.1566 & $\begin{array}{l}\text { B-type procyanidin dimer } \\
\text { digallateb }\end{array}$ & + & + \\
\hline 52 & 31.88 & & Quercetin*c & + & + & $\begin{array}{l}\text { Hydrolysable } \\
\text { tannins and } \\
\text { derivatives }\end{array}$ & & & & + & + \\
\hline 53 & 32.46 & 345.0608 & Quercetin-3,6-dimethyl etherb & + & + & 3 & 1.70 & 331.0648 & Galloyl glucose I & + & + \\
\hline 54 & 32.71 & 315.0514 & Isorhamnetin* & + & + & 6 & 2.72 & 331.0661 & Galloyl glucose II & + & + \\
\hline $\begin{array}{l}\text { Flavonoids- } \\
\text { flavones }\end{array}$ & & & & & & 15 & 16.97 & 483.0816 & Di-O-galloyl-glucose I & + & + \\
\hline 38 & 25.12 & 593.1532 & $\begin{array}{l}\text { Luteolin-7-O-rutinoside } \\
\text { (Scolymoside)*b }\end{array}$ & + & + & 17 & 17.63 & 453.1036 & $\begin{array}{l}\text { Hydroxy-methoxyphenyl-O-(O- } \\
\text { galloyl)-hexose } \mathrm{I}^{\mathrm{b}}\end{array}$ & + & + \\
\hline 41 & 26.00 & 447.0943 & $\begin{array}{l}\text { Luteolin-7-O-glucoside } \\
\text { (Cynaroside)*c }\end{array}$ & + & + & 18 & 17.80 & 483.1122 & $\begin{array}{c}\text { Hydroxy-dimethoxyphenolgalloyl- } \\
\text { glucose }^{b}\end{array}$ & + & + \\
\hline 42 & 26.18 & 329.0671 & Tricin I & + & + & 20 & 17.94 & 453.1031 & $\begin{array}{l}\text { Hydroxy-methoxyphenyl-O-(O- } \\
\text { galloyl)-hexose } \mathrm{Ii}^{\mathrm{b}}\end{array}$ & + & + \\
\hline 56 & 35.75 & 285.0405 & Luteolin*c & + & + & 21 & 18.35 & 483.0763 & Di-O-galloyl-glucose II & + & + \\
\hline
\end{tabular}

${ }^{\mathrm{b} C}$ Compounds described here for first time in family Polygonaceae. Several saccharide combinations and conjugation posititons are reported in different plant families (see KNApSAck, Reaxys or SciFinder databases)

${ }^{\circ}$ compounds were previously reported in severalsRumex species.

${ }^{\mathrm{a}} \mathrm{RT}$, retention time;; Exp, experimental

"Identification confirmed by comparison with standards 